\title{
Endoscopic Minimally Invasive Thyroidectomy: A Prospective Proof-of-Concept Study in Humans
}

\author{
Christopher R. McHenry
}

Published online: 24 December 2010

(C) Société Internationale de Chirurgie 2010

Doctors Wilhelm and Metzig describe a transoral endoscopic sublingual bivestibular approach for removal of the thyroid gland that they first trialed in animals to ensure that no vascular or neural structures were affected [1]. The authors report their initial results in eight patients, four of whom had a total thyroidectomy and the other four had a partial thyroidectomy. In contrast to videoscopic removal of the thyroid gland through a thoracic, axillary, or breast approach, all of which have previously been described for the purpose of making the skin incision and scar less visible, the endoscopic transoral approach eliminates the skin incision.

Of the eight patients who underwent a transoral endoscopic thyroidectomy through a sublingual vestibular approach, three patients had to be converted to a standard open approach, two patients developed recurrent laryngeal nerve injury, one of which was permanent, six patients developed paraesthesias of the lower lip related to transient mental nerve injury, and one patient developed a surgical site infection. One of the recurrent laryngeal nerve injuries occurred in a patient who was converted to an open approach prior to dissection of the thyroid gland. The permanent recurrent laryngeal nerve injury that occurred as a result of the transoral approach was attributed to thermal injury from the harmonic scalpel which is used to divide the ligament of Berry in close proximity to the recurrent laryngeal nerve.

C. R. McHenry ( $\square)$

Department of Surgery, MetroHealth Medical Center,

Cleveland, OH, USA

e-mail: cmchenry@metrohealth.org

C. R. McHenry

Department of Surgery, Case School of Medicine,

Cleveland, OH, USA
Although, the transoral endoscopic approach eliminates the skin incision, I am not sure that this approach fulfills the authors' aims of reducing complications and achieving faster recovery. Removal of the thyroid gland through the mouth changes a clean case to a clean contaminated case with an increased risk of infection. The transoral endoscopic approach is also associated with paraesthesias of the lower lip related to transient injury to the mental nerve. It may also be associated with higher risk of recurrent laryngeal nerve injury which may be the result of thermal injury from the harmonic scalpel. The technique will likely have very limited usefulness as evidenced by the fact three of the eight patients had to be converted to a standard open technique. The technique will have to be limited to patients with small glands of a maximum volume of $30-40 \mathrm{ml}$ and small nodules less than or equal to $2 \mathrm{~cm}$ in size. I suspect there will be interest in this approach purely because of its cosmetic appeal, but before such an approach is considered, it is imperative to ensure that it is as safe as the conventional approach.

\section{Reference}

1. Wilhelm T, Metzig A (2010) Endoscopic minimally invasive thyroidectomy (eMIT): a prospective proof-of-concept study in humans. World J Surg. doi:10.1007/s00268-010-0846-0 